\title{
Fixed points in countably Hilbert spaces
}

\author{
Nashat Faried* and Hany A El-Sharkawy
}

${ }^{\text {*Correspondence: }}$

n_faried@hotmail.com

Department of Mathematics,

Faculty of Science, Ain Shams

University, Abbassia, Cairo, 11566, Egypt

\begin{abstract}
Studying fixed points of nonlinear mappings in Hilbert spaces is of paramount importance (see, e.g., (Browder and Petryshyn in J. Math. Anal. Appl. 20:197-228, 1967)). We extend the notions of weakly contractive and asymptotically weakly contractive nonself-mappings defined on a closed convex proper subset of (into) a real Hilbert space to a real countably Hilbert space. Using the notion of metric projection on countably Hilbert spaces, we study iterative methods for approximating fixed points of nonself-maps. Moreover, we prove convergence theorems with estimates of convergence rates. Furthermore, we also establish the stability of the methods with respect to perturbations of the operators and with respect to the perturbations of the constraint sets.
\end{abstract}

\section{Introduction}

Definition 1.1 (Uniformly convex space [2-6]) A normed linear space $E$ is called uniformly convex if for any $\epsilon \in(0,2]$, there exists $\delta=\delta(\epsilon)>0$ such that if $x, y \in E$ with $\|x\|=1$, $\|y\|=1$, and $\|x-y\| \geq \epsilon$, then $\left\|\frac{1}{2}(x+y)\right\| \leq 1-\delta$.

Definition 1.2 (Modulus of convexity [2-6]) Let $E$ be a normed linear space with $\operatorname{dim} E \geq 2$. The modulus of convexity of $E$ is the function $\delta_{E}:(0,2] \rightarrow[0,1]$ defined by

$$
\delta_{E}(\epsilon):=\inf \left\{1-\left\|\frac{x+y}{2}\right\|:\|x\| \leq 1,\|y\| \leq 1 ;\|x-y\| \geq \epsilon\right\}
$$

Definition 1.3 (Uniformly smooth space [2-6]) A normed linear space $E$ is said to be uniformly smooth if whenever given $\epsilon>0$, there exists $\delta>0$ such that if $\|x\|=1$ and $\|y\| \leq \delta$, then

$$
\|x+y\|+\|x-y\|<2+\epsilon\|y\| .
$$

Definition 1.4 (Modulus of smoothness [2-6]) Let $E$ be a normed linear space with $\operatorname{dim} E \geq 2$. The modulus of smoothness of $E$ is the function $\rho_{E}:[0, \infty) \rightarrow[0, \infty)$ defined by

$$
\begin{aligned}
\rho_{E}(\tau) & :=\sup \left\{\frac{\|x+y\|+\|x-y\|}{2}-1:\|x\|=1 ;\|y\|=\tau\right\} \\
& =\sup \left\{\frac{\|x+\tau y\|+\|x-\tau y\|}{2}-1:\|x\|=1=\|y\|\right\} .
\end{aligned}
$$

(c) 2016 Faried and El-Sharkawy. This article is distributed under the terms of the Creative Commons Attribution 4.0 International License (http://creativecommons.org/licenses/by/4.0/), which permits unrestricted use, distribution, and reproduction in any medium, provided you give appropriate credit to the original author(s) and the source, provide a link to the Creative Commons license, and indicate if changes were made. 
Let $K$ be a nonempty convex subset of a real normed linear space $E$. For strict contraction self-mappings of $K$ into itself, with a fixed point in $K$, a well-known iterative method 'the celebrated Picard method' has successfully been employed to approximate such fixed points. If, however, the domain of a mapping is a proper subset of $E$ (and this is the case in several applications) and if it maps $K$ into $E$, this iteration method may not be well defined. In this situation, for Hilbert spaces and uniformly convex uniformly smooth Banach spaces, this problem has been overcome by the introduction of the metric projection in the recursion formulas (see, e.g., [7-9]). The advantage of this is that if $K$ is a nonempty closed convex subset of a Hilbert space $H$ and $P_{K}: H \rightarrow K$ is the metric projection of $H$ onto $K$, then $P_{K}$ is nonexpansive. This fact characterizes Hilbert spaces and unfortunately is not available in general Banach spaces.

Definition 1.5 (Metric projection $[2,7,8,10]$ ) Let $E$ be a real uniformly convex and uniformly smooth Banach space, $K$ be a nonempty proper subset of $E$. The operator $P_{K}: E \rightarrow K$ is called a metric projection operator if it assigns to each $x \in E$ its nearest point $\bar{x} \in K$, which is the solution of the minimization problem

$$
P_{K} x=\bar{x} ; \quad \bar{x}:\|x-\bar{x}\|=\inf _{\xi \in K}\|x-\xi\| .
$$

Our purpose in this paper is to study, in countably Hilbert spaces, the classes of weakly contractive and asymptotically weakly contractive nonself-maps, which are important extensions of the classes of maps studied by Alber and Guerre-Delabriere [7] and by Chidume et al. [10]. Then, assuming the existence of fixed points for maps in our classes of operators and using several results of Alber and Guerre-Delabriere [7], we prove convergence theorems with estimates of the convergence rates. Our theorems give analogue versions of some results of $[7,10]$ in countably Hilbert spaces.

\section{Preliminaries}

Let $K$ be a nonempty proper subset of a real Banach space $E$. A map $A: K \rightarrow K$ is called a strict contraction if there exists $k \in[0,1)$ such that $\|A x-A y\| \leq k\|x-y\|$ for all $x, y \in K$, and $A$ is called nonexpansive if, for arbitrary $x, y \in K,\|A x-A y\| \leq\|x-y\|$. The map $A$ is called asymptotically nonexpansive if, for all $x, y \in K$, we have $\left\|A^{n} x-A^{n} y\right\| \leq k_{n}\|x-y\|$ for all $n \geq 1$, where $\left\{k_{n}\right\}$ is a sequence of real numbers such that $\lim _{n \rightarrow \infty} k_{n}=1$. It is obvious that for asymptotically nonexpansive mappings, we may assume that $k_{n} \geq 1$ and that $k_{i+1} \leq k_{i}$, $i=1,2, \ldots$ (see, e.g., [3]).

A mapping $A$ is called weakly contractive of the class $C_{\psi(t)}$ on a nonempty set $K$ in a Banach space $E$ if there exists a continuous and nondecreasing function $\psi(t)$ defined on $\mathbb{R}^{+}$with $\psi(t)>0$ for all $t \in \mathbb{R}^{+} \backslash\{0\}, \psi(0)=0$, and $\lim _{t \rightarrow \infty} \psi(t)=+\infty$ such that $\|A x-A y\| \leq$ $\|x-y\|-\psi(\|x-y\|)$ for all $x, y \in K$.

Definition 2.1 (Asymptotically weakly contractive [10]) Let $K$ be a nonempty subset of a real Banach space $E$. A mapping $A: K \rightarrow E$ is called asymptotically weakly contractive of class $C_{\psi(t)}$ if there exists a continuous and nondecreasing function $\psi(t)$ defined on $\mathbb{R}^{+}$ such that $\psi$ is positive on $\mathbb{R}^{+} \backslash\{0\}, \psi(0)=0$, and $\lim _{t \rightarrow \infty} \psi(t)=+\infty$, and there exists a real sequence $\left\{k_{n}\right\} \subseteq[1, \infty)$ with $\lim _{n \rightarrow \infty} k_{n}=1$ such that

$$
\left\|A\left(\Pi_{K} A\right)^{n-1} x-A\left(\Pi_{K} A\right)^{n-1} y\right\| \leq k_{n}\|x-y\|-\psi(\|x-y\|) \quad \forall x, y \in K,
$$


where $\Pi_{K}$ is the generalized projection operator in a Banach space $E$, recently introduced by Alber [8], which is an analogue of the metric projection $P_{K}$ in Hilbert spaces.

Definition 2.2 (Countably normed space [11-14]) Two norms $\|\cdot\|_{1}$ and $\|\cdot\|_{2}$ in a linear space $E$ are said to be compatible if, whenever a sequence $\left\{x_{n}\right\}$ in $E$ is Cauchy with respect to both norms and converges to a limit $x \in E$ with respect to one of them, it also converges to the same limit $x$ with respect to the other norm. A linear space $E$ equipped with a countable system of compatible norms $\|\cdot\|_{n}$ is said to be countably normed. Every countably normed linear space becomes a topological linear space when equipped with the topology generated by the neighborhood base consisting of all sets of the form

$$
U_{\mathfrak{f}, \epsilon}=\bigcap\left\{x: x \in E ;\|x\|_{i}<\epsilon, i \in \mathfrak{f}\right\}
$$

for some number $\epsilon>0$ and finite set $\mathfrak{f}$ of indices.

Remark 2.3 ([14]) By considering the new norms $\|x\|_{n}=\max _{i=1}^{n}\|x\|_{i}$ we may assume that the sequence of norms $\left\{\|\cdot\|_{n} ; n=1,2, \ldots\right\}$ is increasing, that is,

$$
\|x\|_{1} \leq\|x\|_{2} \leq \cdots \leq\|x\|_{n} \leq \cdots \quad \forall x \in E .
$$

If $E$ is a countably normed space, the completion of $E$ in the norm $\|\cdot\|_{n}$ is denoted by $E_{n}$. Then, by definition, $E_{n}$ is a Banach space. Also in the light of Remark 2.3, we can assume that $E \subset \cdots \subset E_{n+1} \subset E_{n} \subset \cdots \subset E_{1}$.

Remark 2.4 In the light of Remark 2.3, we can easily see that the topology of a countably normed space is generated by the neighborhood base consisting of all sets of the form $U_{r, \epsilon}=\left\{x: x \in E ;\|x\|_{r}<\epsilon\right\}$ for a positive integer $r$. Moreover, it is obvious that a nonempty subset $K$ of a countably normed space $E$ is bounded in $E$ if and only if $K$ is bounded in each $\|\cdot\|_{i}$.

Proposition 2.5 ([11]) Let $E$ be a countably normed space. Then $E$ is complete if and only if $E=\bigcap_{n=1}^{\infty} E_{n}$.

Each Banach space $E_{n}$ has a dual, which is a Banach space and denoted by $E_{n}^{*}$.

Proposition 2.6 ([11]) The dual of a countably normed space $E$ is given by $E^{*}=\bigcup_{n=1}^{\infty} E_{n}^{*}$, and we have the following inclusions: $E_{1}^{*} \subset \cdots \subset E_{n}^{*} \subset E_{n+1}^{*} \subset \cdots \subset E^{*}$. Moreover, for $f \in E_{n}^{*}$, we have $\|f\|_{n} \geq\|f\|_{n+1}$.

Example 2.7 ([13]) For $1<p<\infty$, the space $\ell^{p+0}:=\bigcap_{q>p} \ell^{q}$ is a countably normed space. In fact, we can easily see that $\ell^{p+0}=\bigcap_{n} \ell^{p_{n}}$ for any choice of a monotonic decreasing sequence $\left\{p_{n}\right\}$ converging to $p$. Using Proposition 2.5 and the fact that $\ell^{p_{n}}$ is a Banach space for every $n$, it is clear now that the countably normed space $\ell^{p+0}$ is complete.

Definition 2.8 (Countably Hilbert space $[11,12]$ ) A linear space $H$ equipped with a countable system of compatible norms $\|\cdot\|_{n}$ is said to be countably Hilbert space if each $\|\cdot\|_{n}$ is an inner product norm and $E$ is complete with respect to its topology. 
Remark 2.9 In the light of Remark 2.3, Proposition 2.5, and Proposition 2.6, we can see that if $H$ is a countably Hilbert space and if the completion of $H$ in the inner product $\langle\cdot, \cdot\rangle_{n}$ is denoted by $H_{n}$, then by definition, each $H_{n}$ is a Hilbert space, hence $H=\bigcap_{n=1}^{\infty} H_{n}$ and $H^{*}=\bigcup_{n=1}^{\infty} H_{n}^{*}$. Where,

$$
H \subset \cdots \subset H_{n+1} \subset H_{n} \subset \cdots \subset H_{1} .
$$

Remark If $\beta=\left(\beta_{n}\right)$ is a sequence of positive numbers, the dual of the Hilbert space $\ell^{2}(\beta):=\left\{x=\sum_{n=1}^{\infty} x_{n} \frac{e_{n}}{\beta_{n}}: \sum_{n=1}^{\infty}\left|x_{i}\right|^{2}<\infty\right\}$ is the Hilbert space $\ell^{2}(\beta)$.

Example 2.10 The space $E:=\bigcap_{i=1}^{\infty} \ell^{2}\left(\beta^{i}\right)$ is a countably Hilbert space, where $\beta^{i}=\left(\beta_{n}^{i}\right)_{n \in \mathbb{N}}$ satisfies $\beta_{n}^{i} \leq \beta_{n}^{i+1}$, so that the Hilbert spaces $H_{i}=\ell^{2}\left(\beta^{i}\right)$ follow the inclusion $H_{i+1} \subseteq H_{i}$ for all $i$.

Example The Köthe space $\ell^{2}\left[\left\|e_{n}\right\|_{i}\right]:=\left\{x=\left(x_{n}\right):\|x\|_{i}=\left(\sum_{n=1}^{\infty}\left|x_{n}\right|^{2}\left\|e_{n}\right\|_{i}^{2}\right)^{\frac{1}{2}}<\infty, i \in \mathbb{N}\right\}$ with unit basis identified by $\left(e_{n}\right)$ is an example of a countably Hilbert space $\left(E,\|\cdot\|_{i}\right)_{i=1}^{\infty}$ that has an unconditional basis $\left(e_{n}\right)$ (see [15]).

Let $E$ be a countably normed space. In [13], $E$ is called uniformly convex if $\left(E_{i},\|\cdot\|_{i}\right)$ is uniformly convex for all $i$, that is, if for each $i, \forall \epsilon>0 \exists \delta_{i}(\epsilon)>0$ such that if $x, y \in E_{i}$ with $\|x\|_{i}=1=\|y\|_{i}$ and $\|x-y\|_{i} \geq \epsilon$, then $1-\left\|\frac{x+y}{2}\right\|_{i}>\delta_{i}$. The space $E$ is called uniformly smooth if $\left(E_{i},\|\cdot\|_{i}\right)$ is uniformly smooth for all $i$, that is, if for each $i$ whenever given $\epsilon>0$ there exists $\delta_{i}>0$ such that if $\|x\|_{i}=1$ and $\|y\|_{i} \leq \delta_{i}$, then $\|x+y\|_{i}+\|x-y\|_{i}<$ $2+\epsilon\|y\|_{i}$.

Following these two notions, we see that any countably Hilbert space $E$ is uniformly convex and uniformly smooth because each of its $H_{i}$ is a Hilbert space.

In [13], we proved that if $E$ is a countably normed linear space, then:

(i) $E$ is uniformly convex if and only if for each $i, \delta_{E_{i}}(\epsilon)>0$ for all $\epsilon \in(0,2]$.

(ii) $E$ is uniformly smooth if and only if $\lim _{t \rightarrow 0^{+}} \frac{\rho_{E_{i}}(t)}{t}=0$ for all $i$.

(iii) For each $n$, let $E_{n}$ be the completion of $E$ in the norm $\|\cdot\|_{n}$, and $E_{n}^{*}$ its dual. Then for each $i$, we have: for every $\tau>0$,

$$
\rho_{E_{i}}(\tau)=\sup \left\{\frac{\tau \epsilon}{2}-\delta_{E_{i}^{*}}(\epsilon): 0<\epsilon \leq 2\right\}
$$

(iv) $E$ is uniformly smooth if and only if $E_{i}^{*}$ is uniformly convex for all $i$.

(v) $E$ is uniformly convex if and only if $E_{i}^{*}$ is uniformly smooth for all $i$.

Lemma 2.11 (see $[9,16-18]$ ) Let $\left\{\lambda_{k}\right\}$ be a sequence of nonnegative numbers, and $\left\{\alpha_{k}\right\}$ be a sequence of positive numbers such that $\sum_{n=0}^{\infty} \alpha_{n}=\infty$. If the recursive inequality

$$
\lambda_{n+1} \leq \lambda_{n}-\alpha_{n} \psi\left(\lambda_{n}\right), \quad n=1,2, \ldots
$$

holds, where $\psi(\lambda)$ is a continuous strictly increasing function for all $\lambda \geq 0$ with $\psi(0)=0$, then:

1. $\lambda_{n} \rightarrow 0$ as $n \rightarrow \infty$; 
2. the estimate of convergence rate

$$
\lambda_{n} \leq \Phi^{-1}\left(\Phi\left(\lambda_{1}\right)-\sum_{j=1}^{n-1} \alpha_{j}\right)
$$

is satisfied, where $\Phi$ is defined by $\Phi(t)=\int \frac{d t}{\psi(t)}$, and $\Phi^{-1}$ is the inverse function to $\Phi$.

Lemma 2.12 (see $[9,16-18]$ ) Let $\left\{\alpha_{n}\right\},\left\{\beta_{n}\right\},\left\{\lambda_{n}\right\}$, and $\left\{\gamma_{n}\right\}$ be sequences of nonnegative numbers such that $\left\{\alpha_{n}\right\} \subseteq(0,1], \sum_{n=0}^{\infty} \alpha_{n}=\infty, \sum_{n=0}^{\infty} \beta_{n}<\infty$, and $\frac{\gamma_{n}}{\alpha_{n}} \rightarrow 0$ as $n \rightarrow \infty$. If the recursive inequality

$$
\lambda_{n+1} \leq\left(1+\beta_{n}\right) \lambda_{n}-\alpha_{n} \psi\left(\lambda_{n}\right)+\gamma_{n}, \quad n=1,2, \ldots
$$

is given, where $\psi(\lambda)$ is a continuous and nondecreasing function $\psi(t)$ defined on $\mathbb{R}^{+}$such that $\psi$ is positive on $\mathbb{R}^{+} \backslash\{0\}, \psi(0)=0$, and $\lim _{t \rightarrow \infty} \psi(t)=+\infty$. Then

1. $\lambda_{n} \rightarrow 0$ as $n \rightarrow \infty$;

2. there exists a subsequence $\left\{\lambda_{n_{l}}\right\} \subset\left\{\lambda_{n}\right\}, l=1,2, \ldots$, such that

$$
\begin{aligned}
& \lambda_{n_{l}} \leq \psi^{-1}\left(\frac{1}{\sum_{m=1}^{n_{l}} \alpha_{m}}+\frac{\bar{\gamma}_{n_{l}}}{\alpha_{n_{l}}}\right), \quad \bar{\gamma}_{n_{l}}=\gamma_{n_{l}}+\beta_{n_{l}} M, M>0, \\
& \lambda_{n_{l}+1} \leq \psi^{-1}\left(\frac{1}{\sum_{m=1}^{n_{l}} \alpha_{m}}+\frac{\bar{\gamma}_{n_{l}}}{\alpha_{n_{l}}}\right)+\bar{\gamma}_{n_{l}}, \\
& \lambda_{n} \leq \lambda_{n_{l}+1}-\sum_{m=n_{l}+1}^{n-1} \frac{\alpha_{m}}{\mathcal{A}_{m}}, \quad n_{l}+1 \leq n \leq n_{l+1}, \mathcal{A}_{m}=\sum_{i=1}^{m-1} \alpha_{i}, \\
& \lambda_{n+1} \leq \lambda_{1}-\sum_{m=1}^{n} \frac{\alpha_{m}}{\mathcal{A}_{m}} \leq \lambda_{1}, \quad 1 \leq n \leq n_{1}-1, \\
& 1 \leq n_{1} \leq s_{\max }=\max \left\{s: \sum_{m=1}^{s} \frac{\alpha_{m}}{\mathcal{A}_{m}} \leq \lambda_{1}\right\} .
\end{aligned}
$$

Lemma 2.13 (see $[9,16-18]$ ) If $E$ is a uniformly convex space, $K_{1}, K_{2}$ are nonempty closed convex subsets of $E$, and $\mathcal{H}\left(K_{1}, K_{2}\right) \leq \sigma$, then

$$
\left\|P_{K_{1}} x-P_{K_{2}} x\right\| \leq C_{1} \delta_{E}^{-1}(4 L(d+r) \sigma),
$$

where $r=\|x\|, d=\max \left\{d_{1}, d_{2}\right\}, d_{i}=\operatorname{dist}\left(\theta, K_{i}\right), i=1,2, \theta$ is the origin of $E, C_{1}=2 \max \{1, r+$ $d\}$, and $\mathcal{H}$ is the Hausdorff distance.

A version of the following theorem in countably Hilbert spaces is very important and will be used in the proofs of our main results.

Theorem 2.14 ([13]) Let E be a real uniformly convex complete countably normed space, and $K$ be a nonempty convex proper subset of $E$ such that $K$ is closed in each $E_{i}$. Then the metric projection is well defined on $K$, that is,

$$
\forall x \in E \backslash K \exists ! \bar{x} \in K: \quad\|x-\bar{x}\|_{i}=\inf _{\xi \in K}\|x-\xi\|_{i} \quad \forall i
$$




\section{Main results 'successive approximations in a countably Hilbert space'}

In this section we give new versions for some definitions of $[7,10]$ in countably Hilbert spaces and prove our main theorems.

Definition 3.1 (Weakly contractive) Let $K$ be a nonempty subset of a real countably Hilbert space $E$. A mapping $A: K \rightarrow K$ is called weakly contractive of the class $C_{\psi(t)}$ if there exists a continuous and nondecreasing function $\psi(t)$ defined on $\mathbb{R}^{+}$with $\psi(t)>0$ $\forall t \in \mathbb{R}^{+} \backslash\{0\}, \psi(0)=0$, and $\lim _{t \rightarrow \infty} \psi(t)=+\infty$ such that for each $i$, we have $\|A x-A y\|_{i} \leq$ $\|x-y\|_{i}-\psi\left(\|x-y\|_{i}\right) \forall x, y \in K$.

Definition 3.2 (Asymptotically weakly contractive) Let $K$ be a nonempty subset of a real countably Hilbert space $E$. A mapping $A: K \rightarrow E$ is called asymptotically weakly contractive of class $C_{\psi(t)}$ if there exists a continuous and nondecreasing function $\psi(t)$ defined on $\mathbb{R}^{+}$such that $\psi$ is positive on $\mathbb{R}^{+} \backslash\{0\}, \psi(0)=0$, and $\lim _{t \rightarrow \infty} \psi(t)=+\infty$ and if there exists a real sequence $\left\{k_{n}\right\}: k_{n} \geq 1$ with $\lim _{n \rightarrow \infty} k_{n}=1$ such that for each $i$, we have

$$
\left\|A\left(P_{K} A\right)^{n-1} x-A\left(P_{K} A\right)^{n-1} y\right\|_{i} \leq k_{n}\|x-y\|_{i}-\psi\left(\|x-y\|_{i}\right) \quad \forall x, y \in K .
$$

Theorem 3.3 Let $K$ be a nonempty convex subset of a real countably Hilbert space $E=$ $\bigcap H_{n}$ such that $K$ is closed in each $H_{n}$, and $A: K \rightarrow E$ be a weakly contractive map of the class $C_{\psi(t)}$. Suppose that $F(A) \neq \emptyset$ and for $x_{1} \in K$ consider the iteration $x_{n+1}=P_{K} A x_{n}, n \geq 1$. Then $\left\{x_{n}\right\}$ and $\left\{A x_{n}\right\}$ are bounded in $E,\left\{x_{n}\right\}$ strongly converges to some point $x^{*} \in F(A)$, and the estimate

$$
\left\|x_{n}-x^{*}\right\|_{i} \leq \Phi^{-1}\left(\Phi\left(\left\|x_{1}-x^{*}\right\|_{i}-(n-1)\right)\right)
$$

is satisfied for each $i$, where $\Phi$ is defined by $\Phi(t)=\int \frac{d t}{\psi(t)}$.

Proof Since $P_{K}$ is nonexpansive in each $H_{i}$ and $A$ is weakly contractive, for each $i$, we have

$$
\begin{aligned}
\left\|x_{n+1}-x^{*}\right\|_{i} & =\left\|P_{K} A x_{n}-P_{K} A x^{*}\right\|_{i} \\
& \leq\left\|A x_{n}-A x^{*}\right\|_{i} \\
& \leq\left\|x_{n}-x^{*}\right\|_{i}-\psi\left(\left\|x_{n}-x^{*}\right\|_{i}\right) \\
& \leq\left\|x_{n}-x^{*}\right\|_{i} .
\end{aligned}
$$

Hence, for each $i$, we get by induction $\left\|x_{n}-x^{*}\right\|_{i} \leq\left\|x_{1}-x^{*}\right\|_{i}$. Since $x_{1}$ and $x^{*}$ are fixed, this implies that $\left\|x_{n}\right\|_{i} \leq\left\|x_{1}\right\|_{i}+2\left\|x^{*}\right\|_{i}=R_{i}$; therefore, the sequence $\left\{x_{n}\right\}$ is bounded in each $H_{i}$, so it is bounded in $E$.

From (9) it follows that $\left\|A x_{n}\right\|_{i}-\left\|A x^{*}\right\|_{i} \leq\left\|x_{n}\right\|_{i}+\left\|x^{*}\right\|_{i} \leq\left\|x_{1}\right\|_{i}+2\left\|x^{*}\right\|_{i}+\left\|x^{*}\right\|_{i}$. Since $A x^{*}=x^{*}$, we have $\left\|A x_{n}\right\|_{i} \leq\left\|x_{1}\right\|_{i}+4\left\|x^{*}\right\|_{i}=R_{i}^{\prime}$, and therefore $\left\{A x_{n}\right\}$ is bounded.

By (9), for each $i$, the sequence of positive numbers $\left\{\lambda_{n}^{i}\right\}$ defined by $\lambda_{n}^{i}=\left\|x_{n}-x^{*}\right\|_{i}$ satisfies $\lambda_{n+1}^{i} \leq \lambda_{n}^{i}-\psi\left(\lambda_{n}^{i}\right)$. This implies that for each $i$, the sequence $\left\{\lambda_{n}^{i}\right\}$ is nonincreasing and bounded below by 0 , and thus it converges to some $\lambda^{i}$ such that $0 \leq-\psi\left(\lambda^{i}\right) \leq 0$. By the hypothesis on $\psi$ we have that $\lambda^{i}=0$ for all $i$; hence, for each $i, \lambda_{n}^{i} \rightarrow 0$ as $n \rightarrow \infty$. Further, 
by Lemma 2.11 we have the estimate

$$
\lambda_{n}^{i} \leq \Phi^{-1}\left(\Phi\left(\lambda_{1}^{i}\right)-(n-1)\right) \quad \forall n \geq 1
$$

Now, we present stability theorems for the perturbed approximations. First, we study the iterative method with perturbed operators $A_{n}: K \rightarrow E$.

Theorem 3.4 Let $K$ be a nonempty convex subset of a real countably Hilbert space $E$ such that $K$ is closed in each $H_{n}, A: K \rightarrow E$ be a weakly contractive map of the class $C_{\psi(t)}$, and $x^{*} \in K$ be its fixed point. Suppose that there exist sequences of positive numbers $\left\{\delta_{n}\right\}$ and $\left\{h_{n}\right\}$ converging to 0 as $n \rightarrow \infty$ and a finite positive function $g(t)$ defined on $\mathbb{R}^{+}$such that for each $i$ and for all $n \geq 1,\left\|A_{n} v-A v\right\|_{i} \leq h_{n} g\left(\|v\|_{i}\right)+\delta_{n}$. If the iteration $y_{n+1}=P_{K} A_{n} y_{n}$, $n \geq 1$, starting at arbitrary $y_{0} \in K$ is bounded, say by $C$, then it converges in norm to the point $x^{*}$. There exists a subsequence $\left\{y_{n_{l}}\right\}$ of $\left\{y_{n}\right\}, l \geq 1$, such that for each $i$,

$$
\begin{aligned}
& \left\|y_{n_{l}}-x^{*}\right\|_{i} \leq \psi^{-1}\left(\frac{1}{n_{l}}+D h_{n_{l}}+\gamma_{n_{l}}\right), \quad D=g(C), \\
& \left\|y_{n_{l}+1}-x^{*}\right\|_{i} \leq \psi^{-1}\left(\frac{1}{n_{l}}+D h_{n_{l}}+\gamma_{n_{l}}\right)+D h_{n_{l}}+\gamma_{n_{l}}, \\
& \left\|y_{n}-x^{*}\right\|_{i} \leq\left\|y_{n_{l}+1}-x^{*}\right\|_{i}-\sum_{n_{l}+1}^{n-1} \frac{1}{m}, \quad n_{l}+1 \leq n \leq n_{l+1}, \\
& \left\|y_{n+1}-x^{*}\right\|_{i} \leq\left\|y_{1}-x^{*}\right\|_{i}-\sum_{1}^{n} \frac{1}{m} \leq\left\|y_{1}-x^{*}\right\|_{i}, \quad 1 \leq n \leq n_{l}-1, \\
& 1 \leq n_{1} \leq s_{\max }=\max \left\{s: \sum_{1}^{s} \frac{1}{m} \leq\left\|y_{1}-x^{*}\right\|_{i}\right\} .
\end{aligned}
$$

Proof Since the metric projection $P_{K}$ is nonexpansive in each $H_{i}$, it follows that

$$
\begin{aligned}
\left\|y_{n+1}-x^{*}\right\|_{i} & =\left\|P_{K} A_{n} y_{n}-P_{K} A x^{*}\right\|_{i} \\
& \leq\left\|A_{n} y_{n}-A x^{*}\right\|_{i} \\
& \leq\left\|A y_{n}-A x^{*}\right\|_{i}+\left\|A_{n} y_{n}-A y_{n}\right\|_{i} \\
& \leq\left\|y_{n}-x^{*}\right\|_{i}-\psi\left(\left\|y_{n}-x^{*}\right\|_{i}\right)+\gamma_{n}^{i},
\end{aligned}
$$

where $0 \leq \gamma_{n}^{i}=\left\|A_{n} y_{n}-A y_{n}\right\|_{i} \leq D h_{n}+\delta_{n} \rightarrow 0$.

Thus, the sequence of positive numbers defined by $\lambda_{n}^{i}=\left\|y_{n}-x^{*}\right\|_{i}, n \geq 1$, satisfies the recursive inequality $\lambda_{n+1}^{i} \leq \lambda_{n}^{i}-\psi\left(\lambda_{n}^{i}\right)+\gamma_{n}^{i}$. Then the assertion $\lambda_{n}^{i}=\left\|y_{n}-x^{*}\right\|_{i} \rightarrow 0$ as $n \rightarrow$ $\infty$ and estimates (11)-(15) follow from Lemma 2.12 with $\alpha_{i}=1$ and $\beta_{i}=0$ for all $i \geq 1$.

Let us suppose that, instead of an exact set $K$, we have a sequence of perturbed sets $K_{n} \subset E, n \geq 1$, such that the Hausdorff distance $\mathcal{H}\left(K_{n}, K\right) \leq \sigma_{n}$, that is, ' $\mathcal{H}\left(K_{n}, K\right)$ tends to 0 as $n$ tends to $\infty$ '. Let $D(A)$ be any domain for the operator $A$ that contains both $K$ and the perturbed sets $K_{n}$ and such that the Hausdorff distance ' $\mathcal{H}\left(K_{n}, K\right)$ tends to 0 as $n$ tends to $\infty$ ' 
Theorem 3.5 Let $K \subset D(A), K_{n} \subset D(A), n \geq 1$, be nonempty convex sets in a real countably Hilbert space $E$ such that $K$ and $K_{n}$ are closed in each component $H_{n}$ and $\mathcal{H}\left(K_{n}, K\right) \leq \sigma_{n}$, let $A: D(A) \rightarrow$ E be a weakly contractive map of the class $C_{\psi(t)}$ with strictly increasing function $\psi(t)$, and $x^{*} \in K$ be its fixed point. If $\sigma_{n} \rightarrow 0$ as $n \rightarrow \infty$, then the iteration $z_{n+1}=P_{K_{n+1}} A z_{n}$, $n \geq 1$, starting at arbitrary $z_{1} \in K_{1}$ converges in norm to $x^{*}$. There exist a constant $C>0$ and a subsequence $\left\{z_{n_{l}}\right\}$ of $\left\{z_{n}\right\}, l=1,2, \ldots$, such that for each $i$,

$$
\begin{aligned}
& \left\|z_{n_{l}}-x^{*}\right\|_{i} \leq \psi^{-1}\left(\frac{1}{n_{l}}+C \sqrt{\sigma_{n_{l}+1}}\right), \\
& \left\|z_{n_{l}+1}-x^{*}\right\|_{i} \leq \psi^{-1}\left(\frac{1}{n_{l}}+C \sqrt{\sigma_{n_{l}}}\right)+\sqrt{\sigma_{n_{l}}}, \\
& \left\|z_{n}-x^{*}\right\|_{i} \leq\left\|z_{n_{l}+1}-x^{*}\right\|_{i}-\sum_{n_{l}+1}^{n-1} \frac{1}{m}, \quad n_{l}+1 \leq n \leq n_{l+1}, \\
& \left\|z_{n+1}-x^{*}\right\|_{i} \leq\left\|z_{1}-x^{*}\right\|_{i}-\sum_{1}^{n} \frac{1}{m} \leq\left\|z_{1}-x^{*}\right\|_{i}, \quad 1 \leq n \leq n_{l}-1, \\
& 1 \leq n_{1} \leq s_{\max }=\max \left\{s: \sum_{1}^{s} \frac{1}{m} \leq\left\|z_{1}-x^{*}\right\|_{i}\right\} .
\end{aligned}
$$

Proof For each $i$ and all $n \geq 1$, we have

$$
\begin{aligned}
\left\|z_{n+1}-x^{*}\right\|_{i} & =\|P_{K_{n+1}} A z_{n}-\underbrace{A x^{*}}_{=P_{K} x^{*}}\|_{i} \\
& \leq\left\|P_{K_{n+1}} A z_{n}-P_{K_{n+1}} A x^{*}\right\|_{i}+\left\|P_{K_{n+1}} A x^{*}-P_{K} x^{*}\right\|_{i} .
\end{aligned}
$$

Since the metric projection operator is nonexpansive on each closed convex set $K_{n}$, we have

$$
\left\|z_{n+1}-x^{*}\right\|_{i} \leq\left\|A z_{n}-A x^{*}\right\|_{i}+\left\|P_{K_{n+1}} A x^{*}-P_{K} x^{*}\right\|_{i}
$$

By Lemma 2.13, if $\mathcal{H}\left(K_{n+1}, K\right) \leq \sigma_{n+1}$, then there exist positive constants $C_{1} \geq 0$ and $C_{2} \geq 0$ : $\left\|P_{K_{n+1}} x^{*}-P_{K} x^{*}\right\| \leq C_{1} \delta_{E}^{-1}\left(C_{2} \sigma_{n+1}\right)$. Since $\frac{\epsilon^{2}}{8} \leq \delta_{E}(\epsilon)$, that is, $\delta_{E}^{-1}(\grave{\epsilon}) \leq c_{3} \sqrt{\grave{\epsilon}}$, and thus, for the fixed point $x^{*} \in E$, there exists a constant $C>0$ such that

$$
\left\|P_{K_{n+1}} x^{*}-P_{K} x^{*}\right\|_{i} \leq C \sqrt{\sigma_{n+1}}
$$

Since $A$ is weakly contractive, using (23) in (22), we get

$$
\left\|z_{n+1}-x^{*}\right\|_{i} \leq\left\|z_{n}-x^{*}\right\|_{i}-\psi\left(\left\|z_{n}-x^{*}\right\|_{i}\right)+C \sqrt{\sigma_{n+1}} .
$$

Thus, the sequence of positive numbers $\lambda_{n}^{i}:=\left\|z_{n}-x^{*}\right\|_{i}, n \geq 1$, satisfies

$$
\lambda_{n+1}^{i} \leq \lambda_{n}^{i}-\psi\left(\lambda_{n}^{i}\right)+C \sqrt{\sigma_{n+1}}
$$

Since by assumption $\sigma_{n+1} \rightarrow 0$ as $n \rightarrow \infty$, all the conditions of Lemma 2.12 are satisfied with $\alpha_{i}=1$ and $\beta_{i}=0$ for all $i \geq 1$. Thus, $\lambda_{n}^{i} \rightarrow 0$ as $n \rightarrow \infty$, that is, the sequence $\left\{z_{n}\right\}$ 
converges in norm to $x^{*}$, and estimates (17)-(21) are all satisfied, which completes the proof of the theorem.

Now we work in a system of perturbed operators $A_{n}$ and perturbed sets $K_{n}$ to approximate a fixed point $x^{*}$ of the operator $A$ on $K$.

Theorem 3.6 Let $K \subset D(A), K_{n} \subset D(A), n \geq 1$, be nonempty convex sets in a real countably Hilbert space $E$ such that $K$ and $K_{n}$ are closed in each component $H_{n}, A: D(A) \rightarrow E$ be a weakly contractive map of the class $C_{\psi(t)}$ with strictly increasing function $\psi(t)$, and $x^{*} \in K$ be its fixed point. Assume that, instead of $A$, the sequences $\left\{A_{n}\right\}$ of operators $A_{n}: K_{n} \rightarrow E$, $n \geq 1$, are given. Assume also that there exist sequences of positive numbers $\left\{h_{n}\right\}$ and $\left\{\sigma_{n}\right\}$ converging to 0 as $n \rightarrow \infty$ and a finite positive function $g(t)$ defined on $\mathbb{R}^{+}$such that for all $n \geq 1$ and $t \geq 0$, the Hausdorff distance $\mathcal{H}\left(K_{n}, K\right) \leq \sigma_{n}$, and

$$
\left\|A_{n} v-A v\right\|_{i} \leq h_{n} g\left(\|v\|_{i}\right) \quad \forall v \in K_{n} .
$$

If the iteration $u_{n+1}=P_{K_{n+1}} A_{n} u_{n}, n \geq 1$, starting at arbitrary $u_{1} \in K_{1}$ is bounded, then it converges in norm to the point $x^{*}$. Moreover, there exist constants $C>0$ and $C_{1} \geq 0$ and a subsequence $\left\{u_{n_{l}}\right\} \subset\left\{u_{n}\right\}, l \geq 1$, such that for each $i$,

$$
\left\|u_{n_{l}}-x^{*}\right\|_{i} \leq \psi^{-1}\left(\frac{1}{n_{l}}+C \sqrt{\sigma_{n_{l}+1}}\right)+\psi^{-1}\left(\frac{1}{n_{l}}+C_{1} h_{n_{l}}\right)
$$

Proof Considering the iteration $z_{n}$ of Theorem 3.5 with $z_{1} \in K_{1}$, we get

$$
\left\|u_{n+1}-x^{*}\right\|_{i} \leq\left\|u_{n+1}-z_{n+1}\right\|_{i}+\left\|z_{n+1}-x^{*}\right\|_{i}
$$

where $\left\|z_{n+1}-x^{*}\right\|_{i} \rightarrow 0$ as $n \rightarrow \infty$ and $\sigma_{n} \rightarrow 0$ (by Theorem 3.5).

By assumptions $\left\{u_{n}\right\}$ is bounded; then $\forall n \geq 1 \exists M \geq 0:\left\|u_{n}\right\|_{i} \leq M$. Since $A$ is weakly contractive, then using (25), we get

$$
\begin{aligned}
\left\|u_{n+1}-z_{n+1}\right\|_{i} & =\left\|P_{K_{n+1}} A_{n} u_{n}-P_{K_{n+1}} A z_{n}\right\|_{i} \\
& \leq\left\|A_{n} u_{n}-A z_{n}\right\|_{i} \\
& \leq\left\|A u_{n}-A z_{n}\right\|_{i}+\left\|A_{n} u_{n}-A u_{n}\right\|_{i} \\
& \leq\left\|u_{n}-z_{n}\right\|_{i}-\psi\left(\left\|u_{n}-z_{n}\right\|_{i}\right)+h_{n} g\left(\left\|u_{n}\right\|_{i}\right) \\
& \leq\left\|u_{n}-z_{n}\right\|_{i}-\psi\left(\left\|u_{n}-z_{n}\right\|_{i}\right)+h_{n} \underbrace{g(M)}_{=C_{1}} .
\end{aligned}
$$

Thus, the sequence of positive numbers defined by $\lambda_{n}^{i}=\left\|u_{n}-z_{n}\right\|_{i}, n \geq 1$, satisfies the inequality $\lambda_{n+1}^{i} \leq \lambda_{n}^{i}-\psi\left(\lambda_{n}^{i}\right)+C_{1} h_{n}$. Since $h_{n} \rightarrow 0$ as $n \rightarrow \infty$, we have by Lemma 2.12 that

$$
\lambda_{n}^{i}=\left\|u_{n}-z_{n}\right\|_{i} \rightarrow 0 \quad \text { and } \quad\left\|u_{n_{l}}-z_{n_{l}}\right\|_{i} \leq \psi^{-1}\left(\frac{1}{n_{l}}+C_{1} h_{n_{l}}\right) .
$$

Therefore,

$$
\left\|u_{n}-x^{*}\right\|_{i} \leq\left\|u_{n}-z_{n}\right\|_{i}+\left\|z_{n}-x^{*}\right\|_{i} \rightarrow 0, \quad \text { i.e., } u_{n} \rightarrow x^{*} \text { as } n \rightarrow \infty
$$


and

$$
\begin{aligned}
\left\|u_{n_{l}}-x^{*}\right\|_{i} & \leq\left\|u_{n_{l}}-z_{n_{l}}\right\|_{i}+\left\|z_{n_{l}}-x^{*}\right\|_{i} \\
& \leq \psi^{-1}\left(\frac{1}{n_{l}}+C_{1} h_{n_{l}}\right)+\psi^{-1}\left(\frac{1}{n_{l}}+C \sqrt{\sigma_{n_{l}+1}}\right) .
\end{aligned}
$$

Theorem 3.7 Let $K \subset D(A), K_{n} \subset D(A), n \geq 1$, be nonempty convex sets in a real countably Hilbert space $E$ such that $K$ and $K_{n}$ are closed in each $H_{n}, A: D(A) \rightarrow E$ be a weakly contractive map of the class $C_{\psi(t)}$, and $x^{*} \in K$ be its fixed point. Assume that, instead of $A$, the sequences $\left\{A_{n}\right\}$ of the operators $A_{n}: K_{n} \rightarrow E, n \geq 1$, are given. Assume also that there exist sequences of positive numbers $h_{n}, \beta_{n}, \delta_{n}, \mu_{n}$, and $\sigma_{n}$ and a finite positive function $g(t)$ defined on $\mathbb{R}^{+}$such that for each $i$, for all $n \geq 1$ and $t \geq 0$, the Hausdorff distance $\mathcal{H}\left(K_{n}, K\right) \leq \sigma_{n}$, and

$$
\begin{aligned}
& \left\|A_{n} u-A_{n} v\right\|_{i} \leq\left(1+\beta_{n}\right)\|u-v\|_{i}-\psi_{n}\left(\|u-v\|_{i}\right)+\mu_{n} \quad \forall u, v \in K_{n}, \\
& \left\|A_{n} v-A v\right\|_{i} \leq h_{n} g\left(\|v\|_{i}\right)+\delta_{n} \quad \forall v \in K_{n}, \\
& \left|\psi_{n}(t)-\psi(t)\right| \leq v_{n} \quad \forall t \geq 0 .
\end{aligned}
$$

If $\sum_{n=1}^{\infty} \beta_{n}<\infty$ and $\gamma_{n} \rightarrow 0$, where $\gamma_{n}=h_{n}+\delta_{n}+\mu_{n}+v_{n}+\sqrt{\sigma_{n}}$, then the iteration $u_{n+1}=$ $P_{K_{n+1}} A_{n} u_{n}, n \geq 1$, starting at arbitrary $u_{1} \in K_{1}$ converges in norm to the fixed point $x^{*}$.

Remark 3.8 Observe that (27) for $A_{n}$ is similar to the condition of weak contraction of $A$. At the same time, $(28)$ is a standard condition of proximity between $A_{n}$ and $A$ in each point of $K$.

Proof of Theorem 3.7 Consider the iteration of Theorem 3.5 with $z_{1} \in K_{1}$,

$$
\left\|u_{n+1}-x^{*}\right\|_{i} \leq\left\|u_{n+1}-z_{n+1}\right\|_{i}+\left\|z_{n+1}-x^{*}\right\|_{i}
$$

$$
\text { where, for each } i,\left\|z_{n+1}-x^{*}\right\|_{i} \rightarrow 0 \text { as } n \rightarrow \infty \text { and } \sigma_{n} \rightarrow 0 \text { (Theorem 3.5). }
$$

The claim now is to prove that $\left\|u_{n}-z_{n}\right\|_{i} \rightarrow 0$. Noting that the sequence $\left\{z_{n}\right\}$ is bounded, say by $M$, and following (24), (27), (28), and (29), for all $n \geq 1$, we have

$$
\begin{aligned}
\left\|u_{n+1}-z_{n+1}\right\|_{i}= & \left\|P_{K_{n+1}} A_{n} u_{n}-P_{K_{n+1}} A z_{n}\right\|_{i} \\
\leq & \left\|A_{n} u_{n}-A z_{n}\right\|_{i} \\
\leq & \left\|A_{n} z_{n}-A z_{n}\right\|_{i}+\left\|A_{n} u_{n}-A_{n} z_{n}\right\|_{i} \\
\leq & \left(1+\beta_{n}\right)\left\|u_{n}-z_{n}\right\|_{i}-\underline{\psi_{n}\left(\left\|u_{n}-z_{n}\right\|_{i}\right)}+\mu_{n} \\
& +h_{n} g\left(\left\|z_{n}\right\|_{i}\right)+\delta_{n} \\
\leq & \left(1+\beta_{n}\right)\left\|u_{n}-z_{n}\right\|_{i}+\underline{v_{n}-\psi\left(\left\|u_{n}-z_{n}\right\|_{i}\right)}+\mu_{n} \\
& +\underbrace{h_{n} g\left(\left\|z_{n}\right\|_{i}\right)}_{\leq h_{n} g(M)}+\delta_{n} \\
\leq & \left(1+\beta_{n}\right)\left\|u_{n}-z_{n}\right\|_{i}-\psi\left(\left\|u_{n}-z_{n}\right\|_{i}\right)+\gamma_{n},
\end{aligned}
$$


where $\gamma_{n}=\mu_{n}+v_{n}+\delta_{n}+C h_{n}$ and $C=g(M)$. Thus, the positive numbers $\left\{\lambda_{n}^{i}\right\}_{n \geq 1}$ defined by $\lambda_{n}^{i}=\left\|u_{n}-z_{n}\right\|_{i}$ satisfy the inequality

$$
\lambda_{n+1}^{i} \leq\left(1+\beta_{n}\right) \lambda_{n}^{i}-\psi\left(\lambda_{n}^{i}\right)+\gamma_{n}
$$

Since the sequence $\left\{\beta_{n}\right\}$ is summable, that is, $\sum_{n=1}^{\infty} \beta_{n}<\infty$, and hence $\beta_{n} \rightarrow 0$, we can see that the convergence conditions of $\lambda_{n}^{i}$ given by Lemma 2.12 and (32) are the same. Therefore, $\lambda_{n}^{i}=\left\|u_{n}-z_{n}\right\|_{i} \rightarrow 0$ as $n \rightarrow \infty$ and $\gamma_{n} \rightarrow 0$, which proves the claim. Now, using (30) and (31), we conclude that $\lim _{n \rightarrow \infty}\left\|u_{n}-x^{*}\right\|_{i}=0$.

Theorem 3.9 Let $K$ be a nonempty convex subset of a real countably Hilbert space $E$ such that $K$ is closed in each $H_{n}, A: K \rightarrow E$ be an asymptotically weakly contractive map of class $C_{\psi(t)}$ with $\left\{k_{n}\right\} \subseteq[1, \infty)$ such that $\sum_{n=1}^{\infty}\left(k_{n}-1\right)<\infty$, and let $x^{*} \in F(A)$. For $x_{1} \in K$, consider the sequence $\left\{x_{n}\right\}$ defined by

$$
x_{n+1}=\left(P_{K} A\right)^{n} x_{n}, \quad n \geq 1 .
$$

Then $\left\{x_{n}\right\}$ converges strongly to $x^{*} \in F(A)$.

Proof Considering (33) and that $P_{K}$ is nonexpansive and $A$ is asymptotically weakly contractive, set $\beta_{n}=k_{n}-1$. Then, for each $i$, we have

$$
\begin{aligned}
\left\|x_{n+1}-x^{*}\right\|_{i} & =\left\|\left(P_{K} A\right)^{n} x_{n}-\left(P_{K} A\right)^{n} x^{*}\right\|_{i} \\
& \leq\left\|A\left(P_{K} A\right)^{n-1} x_{n}-A\left(P_{K} A\right)^{n-1} x^{*}\right\|_{i} \\
& \leq k_{n}\left\|x_{n}-x^{*}\right\|_{i}-\psi\left(\left\|x_{n}-x^{*}\right\|_{i}\right) \\
& =\left(1+\beta_{n}\right)\left\|x_{n}-x^{*}\right\|_{i}-\psi\left(\left\|x_{n}-x^{*}\right\|_{i}\right) \\
& \leq \exp \left(\sum_{j=1}^{n} \beta_{j}\right)\left\|x_{1}-x^{*}\right\|_{i},
\end{aligned}
$$

so that $\left\|x_{n}-x^{*}\right\|_{i}$ is bounded. If we now set $\lambda_{n}^{i}:=\left\|x_{n}-x^{*}\right\|_{i}$, then Lemma 2.12 and (34) imply $\lim _{n \rightarrow \infty}\left\|x_{n}-x^{*}\right\|_{i}=0$ for all $i$. This completes the proof.

Theorem 3.10 Let $K$ be a nonempty convex subset of a real countably Hilbert space $E$ such that $K$ is closed in each $H_{n}$, let $A: K \rightarrow E$ be a map such that $A\left(P_{K} A\right)^{n-1}$ is bounded and $P_{K} A: K \rightarrow K$ is asymptotically weakly contractive of the class $C_{\psi(t)}$ with $\left\{k_{n}\right\} \subseteq[1, \infty)$ such that $\sum_{n=1}^{\infty}\left(k_{n}-1\right)<\infty$, and let $x^{*} \in F(A)$. Consider the perturbed operators $A_{n}: K \rightarrow E$. Suppose that there exist sequences of positive numbers $\left\{\delta_{n}\right\}$ and $\left\{h_{n}\right\}$ converging to 0 as $n \rightarrow \infty$ and a finite positive function $g(t)$ defined on $\mathbb{R}^{+}$such that for all $n \geq 1$,

$$
\left\|A_{n}\left(P_{K} A_{n}\right)^{n-1} v-A\left(P_{K} A\right)^{n-1} v\right\|_{i} \leq h_{n} g\left(\|v\|_{i}\right)+\delta_{n} \quad \forall v \in K .
$$

If the iteration $y_{n+1}=\left(P_{K} A_{n}\right)^{n} y_{n}, n \geq 1$, starting at arbitrary $y_{1} \in K$ is bounded or $\lim _{n \rightarrow \infty}\left\|\left(P_{K} A_{n}\right)^{n} y_{n}-\left(P_{K} A\right)^{n} y_{n}\right\|_{i}=0$, then it converges to the point $x^{*}$. Moreover, there exists a subsequence $\left\{y_{n_{l}}\right\} \subset\left\{y_{n}\right\}, l \geq 1$, such that

$$
\left\|y_{n_{l}}-x^{*}\right\|_{i} \leq \psi^{-1}\left(\frac{1}{n_{l}}+\bar{\gamma}_{n_{l}}\right),
$$




$$
\begin{aligned}
& \left\|y_{n_{l}+1}-x^{*}\right\|_{i} \leq \psi^{-1}\left(\frac{1}{n_{l}}+\bar{\gamma}_{n_{l}}\right)+\bar{\gamma}_{n_{l}}, \\
& \left\|y_{n}-x^{*}\right\|_{i} \leq\left\|y_{n_{l}+1}-x^{*}\right\|_{i}-\sum_{n_{l}+1}^{n-1} \frac{1}{m}, \quad n_{l}+1 \leq n \leq n_{l+1}, \\
& \left\|y_{n+1}-x^{*}\right\|_{i} \leq\left\|y_{1}-x^{*}\right\|_{i}-\sum_{1}^{n} \frac{1}{m} \leq\left\|y_{1}-x^{*}\right\|_{i}, \quad 1 \leq n \leq n_{l}-1, \\
& 1 \leq n_{1} \leq s_{\max }=\max \left\{s: \sum_{1}^{s} \frac{1}{m} \leq\left\|y_{1}-x^{*}\right\|_{i}\right\} .
\end{aligned}
$$

Proof Set $\beta_{n}:=k_{n}-1$. From the iteration and property of $P_{K} A$ we get

$$
\begin{aligned}
\left\|y_{n+1}-x^{*}\right\|_{i} & =\left\|\left(P_{K} A_{n}\right)^{n} y_{n}-\left(P_{K} A\right)^{n} x^{*}\right\|_{i} \\
& \leq\left\|\left(P_{K} A\right)^{n} y_{n}-\left(P_{K} A\right)^{n} x^{*}\right\|_{i}+\left\|\left(P_{K} A_{n}\right)^{n} y_{n}-\left(P_{K} A\right)^{n} y_{n}\right\|_{i} \\
& \leq k_{n}\left\|y_{n}-x^{*}\right\|_{i}-\psi\left(\left\|y_{n}-x^{*}\right\|_{i}\right)+\left\|\left(P_{K} A_{n}\right)^{n} y_{n}-\left(P_{K} A\right)^{n} y_{n}\right\|_{i} .
\end{aligned}
$$

(i) Assume that the given iteration starting at arbitrary $y_{1} \in K$ is bounded, say by $M>0$; then $\left\{A\left(P_{K} A\right)^{n-1} y_{n}\right\}$ is bounded, and hence, using (35), we get that $\left\{A_{n}\left(P_{K} A_{n}\right)^{n-1} y_{n}\right\}$ is bounded. Thus, by the nonexpansive property of $P_{K}$ in each $H_{i}$ and (35) we get $\left\|\left(P_{K} A_{n}\right)^{n} y_{n}-\left(P_{K} A\right)^{n} y_{n}\right\|_{i} \rightarrow 0$ as $h_{n}, \delta_{n} \rightarrow 0$. Therefore, by (41) all the conditions of Lemma 2.12 are satisfied with $\alpha_{i}=1 \forall i \geq 1$.

(ii) Assume that the assumption $\lim _{n \rightarrow \infty}\left\|\left(P_{K} A_{n}\right)^{n} y_{n}-\left(P_{K} A\right)^{n} y_{n}\right\|_{i}=0$ is satisfied. Then setting $\lambda_{n}^{i}:=\left\|y_{n}-x^{*}\right\|_{i}$, from (41) we get by Lemma 2.12 that the conclusions hold.

\section{Competing interests}

The authors declare that they have no competing interests.

\section{Authors' contributions}

Both authors contributed equally to the manuscripts and read and approved the final manuscripts.

Received: 31 August 2015 Accepted: 15 January 2016 Published online: 27 January 2016

\section{References}

1. Browder, FE, Petryshyn, WV: Construction of fixed points of nonlinear mappings in Hilbert spaces. J. Math. Anal. Appl. 20, 197-228 (1967)

2. Chidume, CE: Applicable Functional Analysis. ICTP Lecture Notes Series (1996)

3. Chidume, CE: Geometric Properties of Banach Spaces and Nonlinear Iterations. Springer, London (2009)

4. Johnson, WB, Lindenstrauss, J: Handbook of the Geometry of Banach Spaces, vol. 1. North-Holland, Amsterdam (2001)

5. Johnson, WB, Lindenstrauss, J: Handbook of the Geometry of Banach Spaces, vol. 2. North-Holland, Amsterdam (2003)

6. Lindenstrauss, J, Tzafriri, L: Classical Banach Spaces II. Springer, Berlin (1979)

7. Alber, Y, Guerre-Delabriere, S: On the projection methods for fixed point problems. Analysis 21(1), 17-39 (2001)

8. Alber, Y: Metric and generalized projection operators in Banach spaces: properties and applications. In: Kartsatos, A (ed.) Theory and Applications of Nonlinear Operators of Monotone and Accretive Type, pp. 15-50. Dekker, New York (1996)

9. Alber, Y: Decomposition theorems in Banach spaces. In: Operator Theory and Its Applications. Fields Institute Communications, vol. 25, pp. 77-93 (2000)

10. Chidume, CE, Khumalo, M, Zegeye, $\mathrm{H}$ : Generalized projection and approximation of fixed points of nonself maps. J. Approx. Theory 120, 242-252 (2003)

11. Becnel, Jj: Countably-normed spaces, their duals, and the Gaussian measure (2005). arXiv:math/0407200v3 [math.FA]

12. Becnel, JJ: Equivalence of topologies and Borel fields for countably-Hilbert spaces. Proc. Am. Math. Soc. 134(2), 581-590 (2006)

13. Faried, N, El-Sharkawy, HA: The projection methods in countably normed spaces. J. Inequal. Appl. 2015(1), 45 (2015). doi:10.1186/s13660-014-0540-0 
14. Kolmogorov, AN, Fomin, SV: Elements of the Theory of Functions and Functional Analysis, vols. 1, 2. Dover, New York (1999)

15. Kondakov, VP: Remarks on the existence of unconditional bases for weighted countably Hilbert spaces and their complemented subspaces. Sib. Math. J. 42(6), 1082-1092 (2001)

16. Alber, $Y$ : The solution of equations and variational inequalities with maximal monotone operators. Sov. Math. Dokl. 20, 871-876 (1979)

17. Alber, Y, Guerre-Delabriere, S: Principle of weakly contractive maps in Hilbert spaces. In: New Results in Operator Theory and Its Applications. Operator Theory: Advances and Applications, vol. 98, pp. 7-22 (1997)

18. Alber, Y, Reich, S: An iterative method for solving a class of nonlinear operator equations in Banach spaces. Panam. Math. J. 4(2), 39-54 (1994)

Submit your manuscript to a SpringerOpen ${ }^{\odot}$ journal and benefit from:

- Convenient online submission

- Rigorous peer review

- Immediate publication on acceptance

- Open access: articles freely available online

- High visibility within the field

- Retaining the copyright to your article 